\section{Ultrasonographic features of the normal filum terminale}

\author{
Myoungae Kwon', Bo-Kyung Je', Doran Hong' ${ }^{1}$ Byung Min Choi ${ }^{2}$ \\ Departments of ${ }^{1}$ Radiology and ${ }^{2}$ Pediatrics, Ansan Hospital, Korea University College of \\ Medicine, Ansan, Korea
}

Purpose: The filum terminale (FT) is a fibrous band that connects the conus medullaris to the posterior body of the coccyx. Considering the advances of ultrasonography (US) technology and improvements in the resolution of US images, we aimed to re-establish the US features of the normal FT in infants younger than 6 months of age.

Methods: We retrospectively reviewed 30 spinal US scans, stored as video clips. The internal structure of the FT and the marginal echogenicity of the FT were assessed, and transverse and longitudinal US were compared.

Results: On US, a central echogenic line was defined in 18 (60\%) normal FTs; however, there was no visible internal structure in 12 cases (40\%). The marginal echogenicity of the FT was hyperechoic in eight cases (27\%) in comparison with the cauda equina and was isoechoic in 22 cases $(73 \%)$. In differentiating the normal FT from the surrounding nerve roots, transverse US was superior in 18 cases (60\%), while longitudinal US was superior in two cases (7\%).

Conclusion: On US, the central canal of the FT was defined in $60 \%$ of normal FTs. Hyperechoic marginal echogenicity and the use of transverse US were helpful in distinguishing the normal FT from the nerve roots of the cauda equina.

Keywords: Ultrasonography; Cauda equina; Infant, newborn; Spinal cord

\section{Introduction}

The filum terminale (FT) is a fibrous band that extends from the conus medullaris to the periosteum of the coccyx, and its functions are to fixate, stabilize, and buffer the distal spinal cord from normal and abnormal cephalic and caudal traction. The cranial intradural part is called the FT internum, and the caudal extradural part is the FT externum [1,2]. The FT is composed largely of loose collagen fibers, small blood vessels, and occasional small nerve fascicles, and contains an extension of the central canal that is lined with ciliated ependymal cells [3-5].

Since the first preliminary study of spinal ultrasonography (US) by Scheible et al. [6], spinal US has been widely used as a screening test in neonates needing evaluation of the spinal cord and/ or the FT. The primary US criterion for an abnormal FT is a thickness $>2 \mathrm{~mm}$, which would lead to suspicion of fibrolipoma involving the FT [6-13]. The US findings of the normal FT have received little attention in the literature; it has been described as a cordlike, echogenic structure surrounded by the echogenic nerve roots of the cauda equina [12]. With advances in US technology, the normal FT can

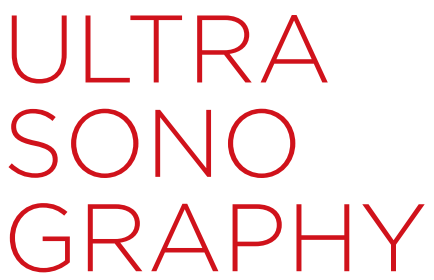

ORIGINAL ARTICLE

https://doi.org/10.14366/usg. 17032 pISSN: 2288-5919 • elSSN: 2288-5943

Ultrasonography 2018;37:129-133

Received: April 13, 2017

Revised: June 8, 2017

Accepted: June 8, 2017

Correspondence to:

Bo-Kyung Je, MD, Department of Radiology, Ansan Hospital, Korea University College of Medicine, 123 Jeokgeum-ro, Danwon-gu, Ansan 15355, Korea

Tel. +82-31-412-5229

Fax. +82-31-412-5224

E-mail: radje@korea.ac.kr

This is an Open Access article distributed under the terms of the Creative Commons Attribution NonCommercial License (http://creativecommons.org/ licenses/by-nc/3.0/) which permits unrestricted noncommercial use, distribution, and reproduction in any medium, provided the original work is properly cited.

Copyright @ 2018 Korean Society of Ultrasound in Medicine (KSUM)

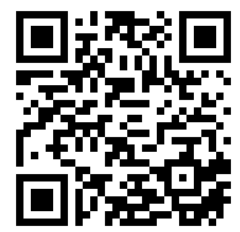

How to cite this article:

Kwon M, Je BK, Hong D, Choi BM Ultrasonographic features of the normal filum terminale. Ultrasonography. 2018 Apr;37(2):129-133. 
be described more precisely than as an echogenic cord. In this study, we aimed to establish the US features of the normal FT in infants younger than 6 months of age using high-resolution US transducers.

\section{Materials and Methods}

This study was approved by the Institutional Review Board of our hospital. The requirement for informed consent was waived, since this was a retrospective study that was limited to previously obtained imaging data.

We reviewed all spinal US exams performed on infants younger than 6 months of age at a single tertiary hospital between January 2016 and December 2016. Spinal US was performed with a 5-12 MHz linear transducer (Logiq E9, GE Healthcare, Milwaukee, WI, USA) in the prone position, or in the left decubitus position, by two radiologists: one with pediatric fellowship training and 16 years of experience, and the other with pediatric fellowship training and 5 years of experience. These two radiologists examined the spinal cord and FT from the coccyx towards the cranium, as far as the mid-thoracic level. In the patients who cooperated with the examination, video clips were obtained in both the transverse and longitudinal directions. We measured the thickness of the FT near the lumbosacral junction in both the transverse and longitudinal directions.

The images were reviewed by two radiologists in consensus: one with pediatric fellowship training and 16 years of experience, and the other with one year of experience. For this study, we enrolled spinal US images with a normal FT and with video clips, which were necessary for an objective retrospective review.

The two readers analyzed the US features of the normal FT focused on 3 points. First, the readers evaluated the visibility of the internal structure, especially the central canal, of the normal FT on each US scan. Second, the readers compared the marginal echogenicity of the FT with that of the nerve roots of the cauda equina and classified the FT as hyperechoic, isoechoic, or hypoechoic. Third, the readers compared the ability of transverse and longitudinal US images to distinguish the presence of a normal FT from the surrounding nerve bundles of the cauda equina, and determined which orientation was superior.

In a review of the patients' medical records, age, gender, and the reason for the US examination were recorded. One-way ANOVA and the $t$ test were used to characterize the relationship between each US feature and the thickness of the FT. The Kruskal-Wallis test was used to determine how each US feature was associated with the patients' age. The chi-square test was used to compare each US feature according to the patients' gender. All statistical analyses were performed using IBM SPSS software ver. 20 (IBM Corp.,
Armonk, NY, USA). P-values of $<0.05$ were considered to indicate statistical significance.

\section{Results}

A total of 114 spinal US scans were performed on infants younger than 6 months of age between January and December 2016. Six patients had a filar cyst, and five showed a ventriculus terminalis at the conus medullaris. Two patients showed low-lying conus medullaris that was located below the L3 vertebral body. There was one fibrolipoma of the FT that was confirmed with magnetic resonance imaging. One patient showed decreased pulsation of the spinal cord. Even though the filar cyst and the ventriculus terminalis were not abnormal findings, but congenital variations, we did not enroll them in this study. Among the remaining 99 patients, a total of 30 spinal US scans (14 males, 16 females; mean age, 12 days; range, 1 to 43 days) included video clips in the transverse and longitudinal directions, and these subjects were ultimately included in the study. These 30 spinal US scans were requested due to concerns over skin dimples located in the midline of the sacrococcygeal area. All dimples were shallow and revealed not to be connected with the spinal canal, which is consistent with a simple sacrococcygeal dimple.

First, the readers evaluated the visibility of the internal structure of the normal FT. In 18 cases (60\%), we could define an echogenic line or a tubular structure with echogenic margins in the center of the FT (Fig. 1). This linear or tubular structure continued from the central echo complex of the spinal cord, which indicated the central canal of the FT. In the remaining 12 cases (40\%), the FT was a hypoechoic cordlike structure without any visible internal structure. When we performed a statistical analysis of the visibility of the central canal according to the patients' age and gender, no factors showed statistical significance. The mean thickness of the FT on the transverse scan was $0.09 \pm 0.02 \mathrm{~mm}$ in patients with a visible internal structure and $0.07 \pm 0.02 \mathrm{~mm}$ in those without a visible internal structure; this difference did not reach statistical significance $(P=0.267)$. The mean thickness of the FT on the longitudinal scan was $0.09 \pm 0.03 \mathrm{~mm}$ in patients with a visible internal structure and $0.08 \pm 0.02 \mathrm{~mm}$ in those without a visible internal structure; this difference likewise did not reach statistical significance $(P=0.105)$.

Second, the readers compared the marginal echogenicity of FT with that of the cauda equina. Eight of the FTs $(27 \%)$ were hyperechoic in comparison to the nerve roots of the cauda equina (Figs. 1, 2). The remaining 22 FTs $(73 \%)$ were isoechoic, and no FT was hypoechoic. When we performed a statistical analysis of the echogenicity of FT according to the patients' age and gender, no factors showed statistical significance. The mean thickness of the $\mathrm{FT}$ on the transverse scan was $0.09 \pm 0.02 \mathrm{~mm}$ in patients with a 
hyperechoic FT and $0.08 \pm 0.02 \mathrm{~mm}$ in those with an isoechoic FT, and this difference did not reach statistical significance $(P=0.895)$. The mean thickness of the FT on the longitudinal scans was $0.10 \pm 0.03 \mathrm{~mm}$ in patients with a hyperechoic FT and $0.08 \pm 0.02$ $\mathrm{mm}$ in those with an isoechoic $\mathrm{FT}$, which likewise did not show statistical significance $(P=0.266)$.

Third, the readers compared transverse US with longitudinal US in terms of the ability to distinguish the normal FT from the surrounding nerve bundles. The transverse scan was superior for differentiating the normal FT in 18 cases (60\%) (Fig. 3). The longitudinal scan was superior in two cases $(7 \%)$, and both directions were rated the same in 10 cases (33\%).

\section{Discussion}

The caudal end of the spinal cord is the conus medullaris, and it continues into the FT, which extends caudally, as a thin and fibrous filament, to insert into the posterior body of $\mathrm{C} 1$. The viscoelasticity of the FT allows the conus medullaris to move slightly during flexion and extension of the spine [14]. For investigations of the spinal cord, FT, cauda equina, and meningeal coverings in the spinal canal, US is regarded as the first-line diagnostic modality in neonates and infants, since at these ages, the posterior vertebral arches are not completely ossified $[4,7-12,15]$.

In the literature, the US features of the spinal cord have been

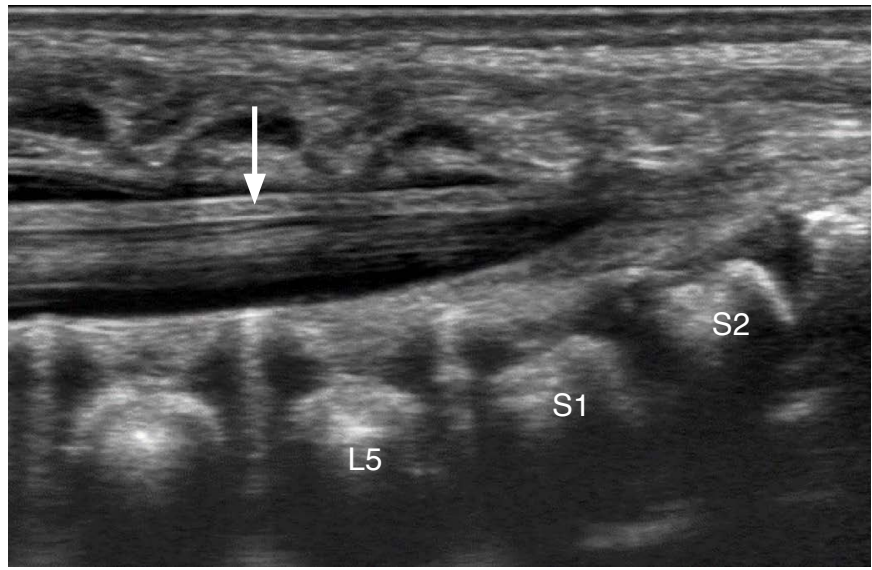

B

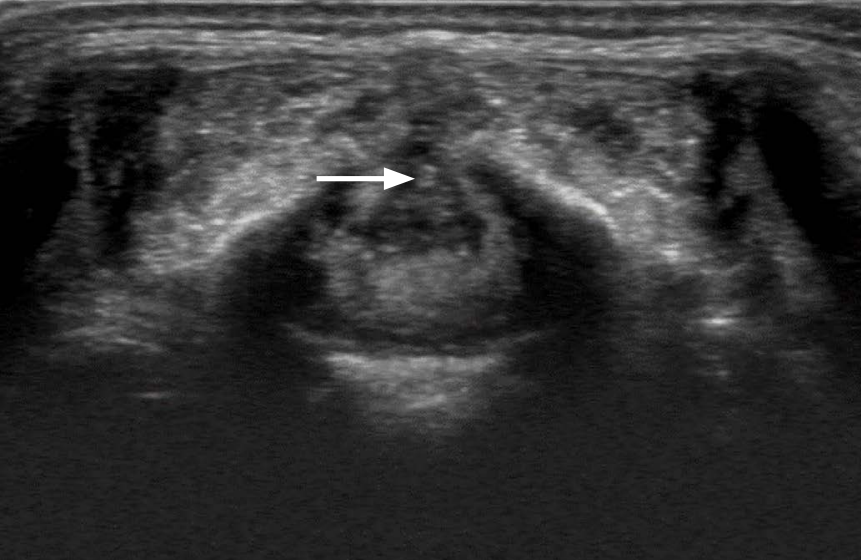

Fig. 1. Spinal ultrasonography in a 1-month-old boy.

A, B. The central canal of the filum terminale (arrows) is visible on transverse (A) and the longitudinal (B) ultrasonography. The marginal echogenicity of the filum terminale is hyperechoic in comparison to the nerve roots of the cauda equina.

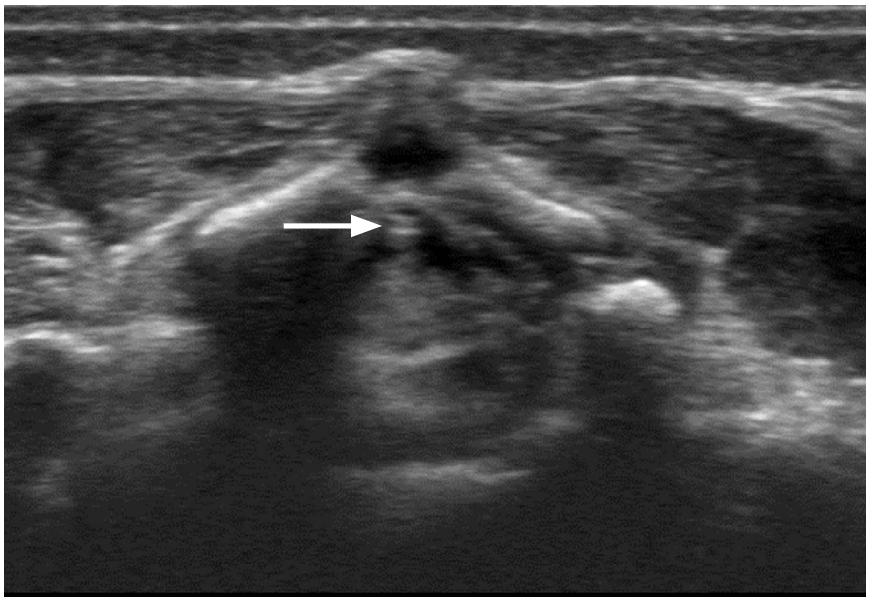

A

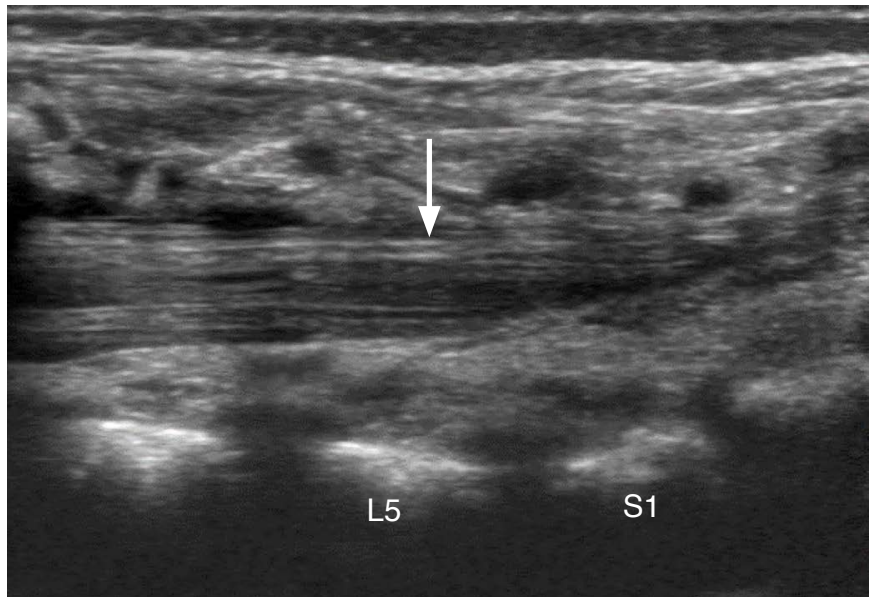

B

Fig. 2. Spinal ultrasonography in an 8-day-old girl.

$A, B$. The marginal echogenicity of the filum terminale (arrows) is hyperechoic in comparison to the nerve roots of the cauda equina on transverse $(A)$ and the longitudinal (B) ultrasonography. 


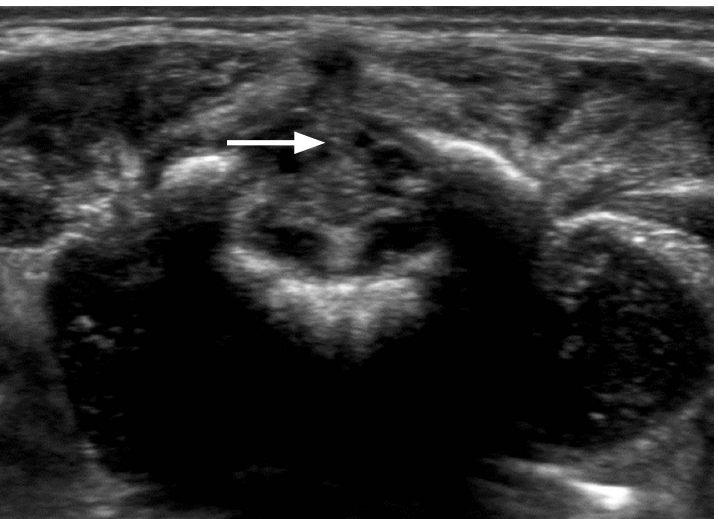

A

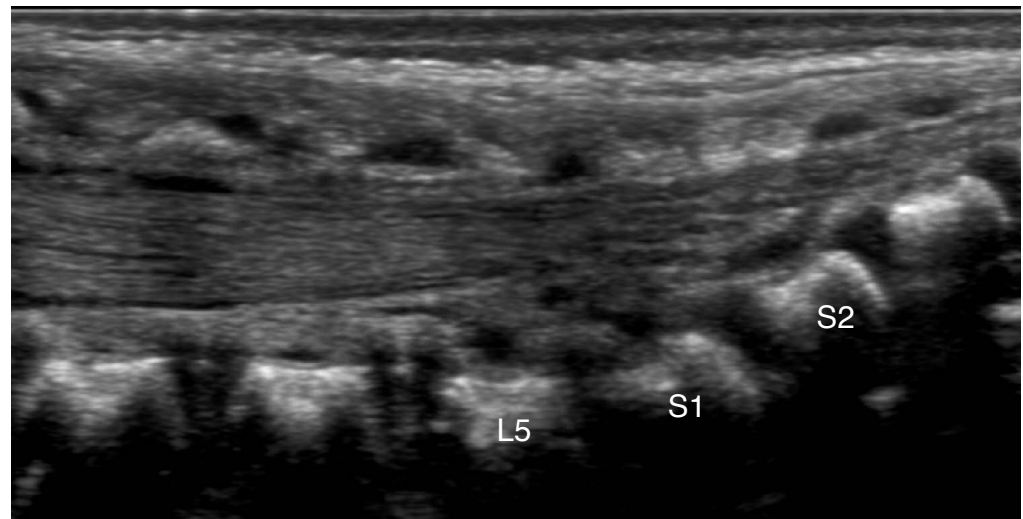

B

Fig. 3. Spinal ultrasonography in a 10-day-old girl.

$A, B$. The filum terminale (arrow) is defined on the transverse ultrasonography (A), while the filum terminale is not separated from the nerve roots of the cauda equina on the longitudinal ultrasonography (B).

described as involving a hypoechoic tubular structure with a central echo complex, while the FT has been described as a hyperechoic cord $[7,10,12]$. Since studies have reported that the FT is not just a simple fibrovascular band, but is also composed of glial cells, connective tissue, and remnants of an ependymal-lined central canal $[5,16-18]$, we tried to determine the ultrastructural US features of the normal FT with the help of current advanced US technology, especially with the increased resolution of US transducers. In our results, the normal FT presented as a hypoechoic cordlike structure on US, and the central canal continuing from the central echo complex of the spinal cord was discernible in $60 \%$ of normal FTs. In the literature, the central canal of the FT has been reported as only being revealed by microscopic evaluations, especially in the FT internum $[3,5,19,20]$.

The pia mater covering the spinal cord continues down to the FT, as was proven via hematoxylin and eosin staining by Fontes et al. [3]. The pia mater extends along each spinal nerve root and becomes continuous with the connective tissue surrounding each spinal nerve [21]. We presumed that the marginal echogenicity of the FT and the cauda equina in our results were due to the pia mater; however, we still could not determine why $27 \%$ of FTs were hyperechoic in comparison to the cauda equina. Nevertheless, the fact that the FT has a more echogenic margin than the cauda equina was helpful for distinguishing the FT from the nerve bundles in cases where it was difficult to identify the normal FT among the congregated or floating nerve bundles of the cauda equina.

We also found that it helped to use transverse US before longitudinal US for distinguishing the FT from the nerve roots, since the transverse scan was superior for differentiating the normal FT in $60 \%$ of cases. We speculate as to the reasons for this result as follows: first, the FT is located in the centermost area of this region, while the nerve roots of the cauda equina spread out. Second, longitudinal scans are not obtained at the exact midline, since it is necessary to perform longitudinal scans in a slightly off-midline plane due to the spinous process.

This study has some limitations. First, the sample size was small; therefore, the statistical analysis of associations between the US features and the patients' information did not show statistical significance. More subjects will be enrolled in a subsequent study. Second, we enrolled US exams that were interpreted as normal. This definition was based on the classic radiological criterion, in which the normal FT is $<2 \mathrm{~mm}$ thick; however, this may not be the gold standard. According to a study by Selcuki et al. [4], a radiologically normal FT may not always confirm a histopathologically normal FT, and Shin et al. [18], in their retrospective study comparing US with magnetic resonance imaging, reported that the optimal US cutoff value for lipomas in the FT was $1.1 \mathrm{~mm}$. A further prospective study is needed to establish updated radiological features of the normal FT.

In conclusion, the central canal of the FT was visible on US in over half of the normal FTs. Even though the majority of FTs were isoechoic with the nerve roots of cauda equina, $27 \%$ were hyperechoic in comparison to the cauda equina, which was helpful to distinguish the FT from the nerve bundles. In addition, transverse US was superior to longitudinal US in distinguishing the normal FT from the nerve roots.

ORCID: Myoungae Kwon: http://orcid.org/0000-0001-8009-3566; Bo-Kyung Je: http://orcid.org/0000-0001-8335-9980; Doran Hong: http://orcid.org/0000-00015777-5630; Byung Min Choi: http://orcid.org/0000-0003-0931-2353 


\section{Conflict of Interest}

No potential conflict of interest relevant to this article was reported.

\section{References}

1. Hansasuta A, Tubbs RS, Oakes WJ. Filum terminale fusion and dural sac termination: study in 27 cadavers. Pediatr Neurosurg 1999;30:176-179.

2. Tubbs RS, Murphy RL, Kelly DR, Lott R, Salter EG, Oakes WJ. The filum terminale externum. J Neurosurg Spine 2005;3:149-152.

3. Fontes RB, Saad F, Soares MS, de Oliveira F, Pinto FC, Liberti EA. Ultrastructural study of the filum terminale and its elastic fibers. Neurosurgery 2006;58:978-984.

4. Selcuki M, Vatansever S, Inan S, Erdemli E, Bagdatoglu C, Polat A. Is a filum terminale with a normal appearance really normal? Childs Nerv Syst 2003;19:3-10.

5. Choi BH, Kim RC, Suzuki M, Choe W. The ventriculus terminalis and filum terminale of the human spinal cord. Hum Pathol 1992;23:916-920.

6. Scheible W, James HE, Leopold GR, Hilton SV. Occult spinal dysraphism in infants: screening with high-resolution real-time ultrasound. Radiology 1983;146:743-746.

7. Dick EA, Patel K, Owens CM, De Bruyn R. Spinal ultrasound in infants. Br J Radiol 2002;75:384-392.

8. Hughes JA, De Bruyn R, Patel K, Thompson D. Evaluation of spinal ultrasound in spinal dysraphism. Clin Radiol 2003;58:227-233.

9. Lowe LH, Johanek AJ, Moore CW. Sonography of the neonatal spine: part 2, Spinal disorders. AJR Am J Roentgenol 2007;188:739744.

10. Lowe LH, Johanek AJ, Moore CW. Sonography of the neonatal spine: part 1, Normal anatomy, imaging pitfalls, and variations that may simulate disorders. AJR Am J Roentgenol 2007;188:733-738.

11. Robinson AJ, Russell S, Rimmer $S$. The value of ultrasonic examination of the lumbar spine in infants with specific reference to cutaneous markers of occult spinal dysraphism. Clin Radiol 2005;60:72-77.

12. Unsinn KM, Geley T, Freund MC, Gassner I. US of the spinal cord in newborns: spectrum of normal findings, variants, congenital anomalies, and acquired diseases. Radiographics 2000;20:923938.

13. Blondiaux E, Katorza E, Rosenblatt J, Nahama-Allouche C, Lenoir M, le Pointe HD, et al. Prenatal US evaluation of the spinal cord using high-frequency linear transducers. Pediatr Radiol 2011;41:374-383.

14. Bui CJ, Tubbs RS, Oakes WJ. Tethered cord syndrome in children: a review. Neurosurg Focus 2007;23:E2.

15. Zieger M, Dorr U, Schulz RD. Pediatric spinal sonography. Part II: Malformations and mass lesions. Pediatr Radiol 1988;18:105-111.

16. Gonzalez-Robles A, Glusman S. The filum terminale of the frog spinal cord: light and electron microscopic observations. Cell Tissue Res 1979;199:519-528.

17. Rosahl SK, Kassem O, Piepgras U, Hellwig D, Samii M. Highresolution constructive interference in steady-state imaging in tethered cord syndrome: technical note. Surg Neurol 2005;63:372374.

18. Shin HJ, Kim MJ, Lee HS, Kim HG, Lee MJ. Optimal filum terminale thickness cutoff value on sonography for lipoma screening in young children. J Ultrasound Med 2015;34:1943-1949.

19. Murthy VS, Deshpande DH. The central canal of the filum terminale in communicating hydrocephalus. J Neurosurg 1980;53:528-532.

20. Gaddam SS, Santhi V, Babu S, Chacko G, Baddukonda RA, Rajshekhar V. Gross and microscopic study of the filum terminale: does the filum contain functional neural elements? J Neurosurg Pediatr 2012;9:86-92.

21. Snell RS. Clinical neuroanatomy. 7th ed. Philadelphia, PA: Wolters Kluwer Health/Lippincott Williams \& Wilkins, 2010. 